\title{
Computational Efficiency and Accuracy for QRS Detection Algorithms on Clinical Long Term Multilead Monitoring
}

\author{
Francisco-Manuel Melgarejo-Meseguer ${ }^{1}$, Estrella Everss Villalba ${ }^{1}$, Jan Široký ${ }^{2}$, \\ Francisco-Javier Gimeno-Blanes ${ }^{3}$, José-Antonio Flores-Yepes ${ }^{4}$, Manuel Blanco-Velasco ${ }^{5}$, \\ José-Luis Rojo-Álvarez ${ }^{6}$, Arcadi García-Alberola ${ }^{1}$ \\ ${ }^{1}$ Hospital Clínico Universitario Virgen de la Arrixaca, Unidad de Arritmias Murcia, Spain \\ ${ }^{2}$ University of West-Bohemian, Pilsen, Czech Republic \\ ${ }^{3}$ Departamento de Comunicaciones. Universidad Miguel Hernández, Elche, Spain \\ ${ }^{4}$ Escuela Politécnica Superior de Orihuela. Universidad Miguel Hernández, Elche, Spain \\ ${ }^{5}$ Departamento de Teoría de la Señal y Comunicaciones. Universidad de Alcalá, Spain \\ ${ }^{6}$ Departamento de Teoría de la Señal y Comunicaciones. Universidad Rey Juan Carlos, Spain.
}

\begin{abstract}
A number of relevant clinical measurements are derived from QRS detection. As a consequence, the fast and accurate calculation becomes a key factor to meet this target, which is especially relevant for the huge amount of beats recorded in the increasingly used long term monitoring. In this paper, we propose several algorithms that present high efficiency and accuracy for the QRS detection in long term, and we benchmark them with some of the most relevant published QRS detection algorithms. All the implemented algorithms were applied to a specifically created gold-standard database. This gold-standard was labelled by expert clinicians, who evaluated manually every beat within 120 records of $48 \mathrm{~h}$ multilead Holter from Hospital Virgen de la Arrixaca of Murcia (Spain). One of our new methods outperformed the others in terms of accuracy and computational efficiency, by using a multilead processing combining OR-function with a new Pan-Tompkins detector. It presented 99.2\% sensitivity, 95.6\% specificity, 97.1\% accuracy, and 77-s processing time for our database. The QRS detection methods used in short term ECG records or traditional 24h Holter can be limited in long term, whereas the proposed multilead processing can provide with better performance in these monitoring scenarios.
\end{abstract}

\section{Introduction}

The main purpose of cardiac monitoring is the detection of cardiac pathologies with transient manifestations or symptoms. The $24 \mathrm{~h}$ holter is the device more frequently used for ECG monitoring in clinical environments. To carry out this test, the patient is subjected to monitoring for twenty-four consecutive hours, while performing their daily activities normally carrying the said device. Research in recent years has proven the long term monitoring (LTM) to be an important prognostic tool. It has been shown that diagnostic indicators are seen more clearly in longer periods. For this reason, recent works are focusing in incremental LTM protocols and systems: 7 days, 15 days, or 21 days. Inside this new scenario, one of the most important challenges is the extraction of different parameters that can be used to improve the accuracy of the diagnosis. The main challenges are in the massive amount of data that the clinician must analyse and validate for each patient, if the traditional protocols and signal processing techniques were to be applied. New protocols and new and more advanced signal processing techniques are required to serve this new framework, and that is where relevant literature is currently focused [1-6].

One of the key parameters to examine the heart behaviour is the heart rate (HR) variation observation, whose analysis supports much heart disease analysis, namely, arrhythmias, transient ischemic episodes, silent myocardial ischemia, and arrhythmic risk assessment of patients, among others [7]. In all cases, and in order to create the heart rate variability signal, the detection of the QRS complex, the R-peak and the time intervals among two consecutive beats, are required.

The objective of this paper is to develop a QRS detection algorithm capable of running with the highest possible computational efficiency and accuracy over LTM records. This goal has been accomplished by using an algorithm that takes into account both the inter-lead and the intra-lead information by adjusting the threshold level and optimizing the computational requirements. Accuracy and computational efficiency of algorithms were tested over, a trusted and solid set of $48 \mathrm{~h}$ holter 
records, previously and carefully labelled by the clinical team from Hospital Universitario Virgen de la Arrixaca (Murcia, Spain). Conventional statistics and ratios were used for comparison: sensitivity, specificity, positive predictive value, and negative predictive value.

The present paper is structured as follows. In Section 2, we first describe the appliances and tools used for signal registering and processing. Second, we elaborate on all algorithms applied for signal processing. In Section 3, we include the different experiments and results of selected methods applied over our database. Finally, Section 4 contains the discussion and conclusions.

\section{Methods}

\subsection{Equipment and algorithm overview}

Existing public and well referenced databases such as MIT-BIH arrhythmia database or QT databases do not contain LTM registers with over 24h signals, so a tailored database was developed for the purpose of this project. We will refer this database as the Long Term Database or LTDB in this paper, which incorporates 48 hours recordings of 17 patients from 5 different hospitals. Two patients exhibited a normal heart function, five had an implanted defibrillator, five presented atrial fibrillation, and the last five suffered from frequent premature ventricular contractions.

Two different Holter recorders were used for the ECG signal registering, namely, the SpiderView, from ELA medical, and SpiderView Plus, from Sorin Group. Sampling frequency was $200 \mathrm{~Hz}$. The number of sensors used for the acquisition were 3 and 12. (i.e., with monitoring periods longer than 24 hours).

The signal processing was structured in four phases: segmentation, for increasing computational efficiency, pre-processing for denoising purposes and initial adjustments, feature extraction for highlighting QRS complexes, and $R$-peaks detection by thresholding for final detection and HR analysis.

Segmentation. Special remark is required at this point with regard to the large size of the signals. A 3-leads signal, sampled at $200 \mathrm{~Hz}$ with 15 bits resolution during 48 consecutive hours creates a single file of $100 \mathrm{MB}$, so, to process it in a standard computer, adequate segmentation for efficient computation is required.

Pre-Procesing incorporated baseline wander, bandpass filtering and detection of signal presence. The bandpass filter was defined with low-high cut-off frequencies of $1 \mathrm{~Hz}-50 \mathrm{~Hz}$. The baseline wander implementation incorporated a polynomial interpolation algorithm.

The presence or absence of the signal was evaluated based on empirical thresholding of classical statistical parameters, such as the standard deviation.

\subsection{Feature signal computation}

Several algorithms were compared while extracting a feature signal for thresholding using Independent Component Analysis (ICA), Principal Component Analysis (PCA), Pan Tompkins (PT), Root Mean Square (RMS), And, Or, Simple Coupling (SC) and Polling (Poll).

ICA decomposition. The ICA method provides the decomposition of the signals in a new set of statistical independent signals. In this technique, it is intended to separate originally related elements, from none related noisy components.

Mathematically, let $\boldsymbol{X}$ be the matrix with recorded multilead signals and let $\boldsymbol{S}$ be the unmixed set of source signals, matrix $\boldsymbol{A}$ represents a linear mixing condition which transforms the original signal set into the recorded one. In matrix form, the mixture and unmixture equations can be denoted as follows,

$$
X=A S \rightarrow S=A^{-1} X=W X
$$

Therefore, the ICA problem focusses on estimating $\boldsymbol{A}$ and $\boldsymbol{S}$ matrices from the $\boldsymbol{X}$ matrix, by applying the condition that the $\boldsymbol{S}$ matrix components must be statistically independent, and the problem can be reformulated to find the $\boldsymbol{W}$ matrix minimizing the $\boldsymbol{S}$ components Gaussianity. For that purpose a FastICA algorithm was applied. The FastICA outputs are disordered and thus the cleaned ECG signal is chosen by measuring the kurtosis [8].

PCA decomposition. The PCA model aims to find a source signal as well, but does not assume the independence in resulting sets, but rather orthogonally. It is assume that one or more final components will be free from all type of noise. To perform this goal, it is necessary to transform a coordinate system of a given dataset in order to increase its variation. Then, the covariance matrix of all leads is calculated and the eigenvectors magnitudes are measured. A final transformation is done according to the highest eigenvector. The PCA output is a dataset where the wanted characteristic ECG is often the first component [9].

PT algorithm. The PT algorithm obtains the characteristic signal by peaking only one lead from the dataset (one with the lowest noise content), filtering with a bandpass filter and applying the following derivative filter:

$$
H(z)=0.1\left(-2 z^{-2}-z^{-1}+z+2 z^{2}\right)
$$

Then, the signal is squared to boost the R-peaks amplitude and median/integration filter is applied with a sliding window. This method uses one single lead, hence, any relevant information from other leads might not be used and therefore limiting the total eventual power of the usage of all existing information [10]. 
RMS algorithm. To improve the PT mentioned disadvantage, we developed the RMS algorithm, which creates a characteristic signal by combining all the leads as

$$
y_{c}(n)=\sqrt{\sum_{l=1}^{L} x^{2}(n, l)}
$$

where $y_{c}$ stands for the characteristic signal, $x(n, l)$ denotes $l$-th lead of the input signal after pre-processing stage, and $L$ is the number of leads.

\subsection{Combinational methods}

In order to enhance noise protection and to take benefit from multiple lead, we developed several combinational methods for obtaining a characteristic signal. The proposed methods are based on a tailored PT algorithm, taking into account the intra-lead information as well as the inter-lead one. The set of characteristic signals is created according to the following equation,

$$
y_{c}\left(n, l_{i}\right)=x_{f}\left(n, l_{i}\right) \sqrt{\prod_{l=1 l \neq l_{i}}^{L} x_{f}(n, l)}
$$

where the $y_{c}\left(n, l_{i}\right)$ is the characteristic signal of $i$-th lead and $x_{f}\left(n, l_{i}\right)$ is the preprocessed signal filtered with the PT filter (2). R-peaks are detected in every characteristic signal and subsequently, the detection results are combined. We have designed four different possibilities, as follows:

1. And. Logic function AND, which reports an Rpeak only when it is detected in all leads in the same time.

2. Poll. If a peak is detected in the majority of leads, it is reported as a good detection.

3. SC. To report an R-peak detection, it must be found in at least two leads.

4. Or. Logical function OR, which reports an Rpeak if it is detected in at least one lead.

While comparing detections in different leads, we implement a tolerance window to take into account the delay between leads. The value of this window was chosen to be $10 \mathrm{~ms}$, a time delay which stands for less than $10 \%$ of the whole R-peak duration.

\subsection{R-peak detection}

Once the feature signal is in place, the R-peak threshold detection can be applied. We neglect the $\mathrm{T}$ peaks by using a double threshold technique. The first threshold acts over the amplitude to detect the peaks and the second one is applied over the time to check the condition imposed by the cardiac refractory period.

\section{Experiments and results}

In order to analyse the behaviour of our algorithm over the most commons configurations of Holters (i.e., two lead, and three leads), we created two subsets using our database: the first one containing recordings with two leads and the second one containing recordings with three leads. We made these subsets using a selection of the leads for every the register of our database. In every experiment described from now on, we specify the subset used.

The figures of merit used were: Sensibility (S), Positive Predictive Value (P), and Accuracy (A).

\subsection{Efficiency improvement}

To optimize the computational efficiency, different signal segmentation strategies were examined. In terms of computational efficiency, the worst case scenario will be the one containing the highest number of leads, and for that reason the 3 leads Holter database was selected to perform this experiment. S, P and A were selected as merit figures. Table 1 shows the optimal segment length for every algorithm. It can be seen that 5 min was the best trade-off for computing time and good performance for several methods.

\subsection{Threshold optimization}

In this experiment we used both data subsets to reach the highest accuracy and independency on parameters of a given signal, and several threshold level calculations were tested. These thresholds were calculated by adding the $\mathrm{x}$ th multiple of signal average value with the y-th multiple of characteristic signal standard deviation. As figure of merit, we used S, P and A. Table 2 shows the mean of the figures of merit for every algorithm using its optimal amplitude threshold.

\begin{tabular}{|c|c|c|c|c|c|}
\hline \multirow{2}{*}{ Method } & \multirow{2}{*}{$\begin{array}{c}\text { Segment } \\
\text { length }\end{array}$} & \multirow{2}{*}{$\begin{array}{c}\text { Computing } \\
\text { time [s] }\end{array}$} & \multicolumn{3}{|c|}{$\begin{array}{c}\text { Statistical measures } \\
\text { [\%] }\end{array}$} \\
\cline { 4 - 6 } & & & $\mathrm{S}$ & $\mathrm{P}$ & $\mathrm{A}$ \\
\hline ICA & $6 \mathrm{~h}$ & 99,44 & 90,48 & 84,80 & 78,99 \\
\hline PCA & $30 \mathrm{~min}$ & 83,71 & 96,79 & 95,89 & 93,00 \\
\hline RMS & $30 \mathrm{~min}$ & 79,18 & 99,07 & 94,21 & 93,48 \\
\hline PT & $2 \mathrm{~h}$ & 76,24 & 99,46 & 95,96 & 95,48 \\
\hline And & 5 min & 99,08 & 96,42 & 95,59 & 99,08 \\
\hline Or & $\mathbf{5}$ min & $\mathbf{7 7 , 3 3}$ & $\mathbf{9 9 , 6 1}$ & $\mathbf{9 5 , 7 8}$ & $\mathbf{9 5 , 4 4}$ \\
\hline SC & 5 min & 77,33 & 99,28 & 96,09 & 95,45 \\
\hline Poll & 5 min & 77,33 & 99,28 & 96,09 & 95,45 \\
\hline
\end{tabular}

Table 1 Computational efficiency optimization 


\begin{tabular}{|c|c|c|c|c|c|c|}
\hline \multirow{2}{*}{ Method } & \multicolumn{6}{|c|}{ Statistical measures [\%] } \\
\cline { 2 - 7 } & \multicolumn{2}{|c|}{ 2-leads database } & \multicolumn{3}{|c|}{ 3 -leads database } \\
\cline { 2 - 7 } & $\mathrm{S}$ & $\mathrm{P}$ & $\mathrm{A}$ & $\mathrm{S}$ & $\mathrm{P}$ & $\mathrm{A}$ \\
\hline Poll & 97,81 & 93,53 & 93,53 & 98,98 & 95,29 & 95,29 \\
\hline Or & $\mathbf{9 9 , 8 4}$ & $\mathbf{9 2 , 6 3}$ & $\mathbf{9 2 , 6 3}$ & $\mathbf{9 9 , 7 8}$ & $\mathbf{9 3 , 2 2}$ & $\mathbf{9 3 , 2 2}$ \\
\hline And & 97,81 & 93,53 & 93,53 & 98,50 & 95,49 & 95,49 \\
\hline SC & 97,81 & 93,53 & 93,53 & 98,39 & 92,94 & 92,94 \\
\hline ICA & 84,23 & 70,53 & 70,53 & 88,56 & 70,53 & 70,53 \\
\hline PT & 98,21 & 88,26 & 88,26 & 98,21 & 88,26 & 88,26 \\
\hline RMS & 98,04 & 89,05 & 89,05 & 98,07 & 89,16 & 89,16 \\
\hline PCA & 95,63 & 84,12 & 84,12 & 95,73 & 85,93 & 85,93 \\
\hline
\end{tabular}

\section{References}

[1] Dagres N, Kottkamp H, et al. Influence of the Duration of Holter Monitoring on the Detection of Arrhythmia Recurrences After Catheter Ablation of Atrial Fibrillation. Intern Jour Cardiol. 2010;139(3):305-306.

[2] Steven D, Rostock T, et al. What is the Real Atrial Fibrillation Burden after Catheter Ablation of Atrial Fibrillation? A Prospective Rhythm Analysis in Pacemaker Patients with Continuous Atrial Monitoring. European Heart Journal. 2008;29(8):1037-1042

[3] Seto E. Cost Comparison Between Telemonitoring and Usual Care of Heart Failure: A Systematic Review. Telemedicine and e-Health. 2008;14(7):679686.

Table 2 Statistical measures for 2 and 3 leads database.

[4] Jabaudon D, Sztajzel J, Sievert K, Landis T, Sztajzel R. Usefulness of Ambulatory 7-Day ECG Monitoring for the Detection of Atrial Fibrillation and Flutter After Acute Stroke and Transient Ischemic Attack. Stroke. 2004;35(7):1647-1651.

\subsection{Optimal R-peak Detection Method}

According to previous experiments, the OR combinational method outperformed the rest of the developed techniques (see Table 2). It standouts in terms of computational time, as well as in detection success.

During this experiment, free parameters tuning processes were performed with different strategies to improve accuracy, with the following results. Low passfilter cut-off frequency of $25 \mathrm{~Hz}$ showed better results than the original $50 \mathrm{~Hz}$. Refractory period of the algorithm was adjusted, as well as key parameters for denoising mechanisms. The optimized algorithm results showed 99,23\% S, 95,63\% specificity, 95,71\% P, 99,23\% negative predictive value, and $97,06 \% \mathrm{~A}$.

\section{Conclusion}

The method defined in this paper, with a suitable segmentation, using the modified QRS detection algorithm defined by PT, and using the complete information present in all available leads combined with an OR function, outperformed all the previously described algorithms over a $48 \mathrm{~h}$ holter signals. Mentioned improvements are two fold, in terms of accuracy and computational efficiency. Further analysis and comparisons over public databases are required for the validations of presented findings.

\section{Acknowledgement}

This work has been partially supported by: TEC201348439-C4-2-R (supported with FEDER fundings), TEC2010-19263, TEC2016-75161-C2-2-R, IPT-20121126-300000,DP12011-27002-C02-01, ACOMP/2013 /018, PI11/02549, and RD06/0014/0017.

[5] Pastor-Pérez F, Manzano-Fernández S, GoyaEsteban R, Pascual-Figal D, Barquero-Pérez O, Rojo Álvarez J et al. Prognostic Significance of LongPeriod Heart Rate Rhythms in Chronic Heart Failure. Circulation Journal. 2012;76(9):2124-2129.

[6] Martínez A, Everss E, Rojo-Álvarez J, Figal D, García-Alberola A. A Systematic Review of the Literature on Home Monitoring for Patients with Heart Failure. J Telemed Telecare. 2006;12(5):234-241.

[7] G. Walraven, Basic Arrhythmias [With Access Code]. AddisonWesley Pub Co Inc, 2010. Available: www.ebook.de/de/product/11024159/gail_walraven basic_arrhythmias_with_access_code.html

[8] Kuzilek JLhotska L. Electrocardiogram Beat Detection Enhancement using Independent Component Analysis. Medical Engineering \& Physics. 2013;35(6):704-711.

[9] Chawla M, Verma H, Kumar V. ECG Modeling and QRS Detection using Principal Component Analysis. 3rd International Conference MEDSIP 2006 Advances in Medical, Signal and Information Processing. 2006.

[10] Pan JTompkins W. A Real-Time QRS Detection Algorithm. IEEE Transactions on Biomedical Engineering. 1985; 32(3):230-236.

Address for correspondence: Francisco-Javier Gimeno-Blanes Innova Bld., Av. Univesidad, s/n, 03202 Elche Alicante, Spain javier.gimeno@umh.es 